\title{
HESS J0632+057: hydrodynamics and non-thermal emission
}

\author{
Valentí Bosch-Ramon, ${ }^{1}$ Maxim V. Barkov, ${ }^{2,3 \star}$ Andrea Mignone ${ }^{4}$ and Pol Bordas ${ }^{5}$ \\ ${ }^{1}$ Departament de Física Quàntica i Astrofísica, Institut de Ciències del Cosmos (ICCUB), \\ ${ }^{2}$ Universitat de Barcelona, IEEC-UB, Martí i Franquès 1, E-08028 Barcelona, Spain \\ ${ }^{3}$ Department of Physics and Astronomy, Purdue University, West Lafayette, IN 47907-2036, USA \\ ${ }^{4}$ Astrophysical Big Bang Laboratory, RIKEN, 351-0198 Saitama, Japan \\ ${ }^{5}$ Dipartimento di Fisica Generale Facolta di Scienze M.F.N., Universita degli Studi di Torino, I-10125 Torino, Italy \\ ${ }^{6}$ Max-planck-Institut für Kernphysik, Saupfercheckweg 1, D-69117 Heidelberg, Germany
}

Accepted 2017 July 28. Received 2017 July 28; in original form 2017 June 20

\begin{abstract}
HESS J0632+057 is an eccentric gamma-ray Be binary that produces non-thermal radio, $\mathrm{X}$ rays, $\mathrm{GeV}$ and very high-energy gamma-rays. The non-thermal emission of HESS J0632+057 is modulated with the orbital period, with a dominant maximum before apastron passage. The nature of the compact object in HESS J0632+057 is not known, although it has been proposed to be a young pulsar as in PSR B1259-63, the only gamma-ray emitting high-mass binary known to host a non-accreting pulsar. In this letter, we present hydrodynamical simulations of HESS J0632+057 in the context of a pulsar and a stellar wind interacting in an eccentric binary, and propose a scenario for the non-thermal phenomenology of the source. In this scenario, the non-thermal activity before and around apastron is linked to the accumulation of non-thermal particles in the vicinity of the binary, and the sudden drop of the emission before apastron is produced by the disruption of the two-wind interaction structure, allowing these particles to escape efficiently. In addition to providing a framework to explain the nonthermal phenomenology of the source, this scenario predicts extended, moving X-ray emitting structures similar to those observed in PSR B1259-63.
\end{abstract}

Key words: hydrodynamics - radiation mechanisms: non-thermal-stars: winds, outflowsgamma-rays: stars - X-rays: binaries.

\section{INTRODUCTION}

The very high-energy (VHE) point-like source HESS J0632+057, detected in Monoceros by HESS (Aharonian et al. 2007), was proposed by Hinton et al. (2009) to be the fourth gamma-ray binary known after PSR B1259-63, LS 5039 and LS I +61 303 (Tavani et al. 1998; Paredes et al. 2000; Aharonian et al. 2005a,b; Albert et al. 2006). The binary nature of HESS J0632+057 was confirmed in X-rays when the orbital period was established in $T \approx 321 \mathrm{~d}$ (Bongiorno et al. 2011) ${ }^{1}$, and Casares et al. (2012) further characterized the system, which consists of a Be star and a compact object of unknown nature, as highly eccentric, $e=0.83$, and has an orbital semi-major axis of $a \approx 4 \times 10^{13} \mathrm{~cm}$.

The X-ray and the VHE light curves of HESS J0632+057 are quite similar. They show a broad maximum, around orbital phases $\psi \sim 0.3-0.4$, between periastron $(\psi=0)$ and apastron passages. Then, both the X-ray and the VHE emission fall sharply right before

\footnotetext{
${ }^{\star}$ E-mail: barmv05@gmail.com

${ }^{1}$ Note that a $T=315 \mathrm{~d}$, consistent with $T=321 \mathrm{~d}$, was obtained by Aliu et al. (2014) using more X-ray data.
}

apastron, around $\psi \sim 0.4-0.5$, and around $\psi \sim 0.6-0.8$ the X-ray and VHE emission present a secondary, less prominent maximum (e.g. Aliu et al. 2014). Emission in GeV has been detected as well, which despite the low statistics is consistent with having a peak before apastron (Li et al. 2017). On the other hand, radio very long baseline interferometry (VLBI) observations at $1.6 \mathrm{GHz}$, probing spatial scales $\sim 10^{15} \sim(10-100) a \mathrm{~cm}$, found a flux decrease around $\psi \sim 0.4-0.5$ (Moldón, Ribó \& Paredes 2011), and a shift in position by 14 mas, or $\sim 21$ au in projected size at $\sim 1.4 \mathrm{kpc}$ distance. The shift was found between two runs $30 \mathrm{~d}$ apart, implying a velocity of $\gtrsim 1.2 \times 10^{8} \mathrm{~cm} \mathrm{~s}^{-1}$. In the second run, a radio component appeared showing extension on scales of $\sim 10$ mas in the opposite direction to that of the position shift.

The system parameters and the non-thermal emission of HESS J0632+057 are similar to those of the gamma-ray binary PSR B1259-63 (see Dubus 2013, and references therein), which may suggest the same nature of the compact object in both sources, a young pulsar non-accreting for most of its orbit (see however, Zamanov, Marti \& Garcia-Hernandez 2017). In this case, one expects a complex physical evolution of the pulsar and the stellar winds as they interact along the very eccentric orbit. Such a complexity is clearly seen, for instance in the hydrodynamical simulations done 
by Barkov \& Bosch-Ramon (2016) of the two-wind interaction for PSR B1259-63, which provided a framework to explain the moving extended X-ray structures on arcsecond scales found by Kargaltsev et al. (2014) and Pavlov et al. (2015).

Motivated by the results of Barkov \& Bosch-Ramon (2016) on PSR B1259-63, and aiming at a deeper exploration of HESS J0632+057, we have carried out relativistic hydrodynamical simulations of the pulsar-stellar wind interaction in HESS J0632+057 on scales $\sim(1-1000) a$ accounting for orbital motion (Section 2). Using the simulation results, we have developed a specific scenario that explains most of the phenomenology of the non-thermal emission of HESS J0632+057 along the orbit, and also predicts transient X-ray emission on $\sim$ arcsecond scales (Section 3).

\section{SIMULATIONS OF THE TWO-WIND COLLISION IN HESS J0632+057}

The relativistic hydrodynamical simulations were done with the PLUTO code $^{2}$ (Mignone et al. 2007) using a simplified threedimensional (3D) geometry in spherical coordinates. PLUTO is a modular Godunov-type code intended mainly for astrophysical applications in multiple spatial dimensions and flows of high Mach numbers. A third-order Runge-Kutta approximation in time, spatial parabolic interpolation and an HLLC Riemann solver were used (Mignone \& Bodo 2005). The simulations were run through the MPI library in the CFCA cluster of the National Astronomical Observatory of Japan and GreatWave FX100 Fujitsu cluster in RIKEN (Japan).

The simulation set-up is similar to the one adopted in Barkov \& Bosch-Ramon (2016) for PSR B1259-63, as it allows one to capture the main physical features of the problem on scales $\sim 1000 a$ in a reasonable computation time-scale. In the case of HESS J0632+057 considered here, the computational domain has a size of $r=[0.62 a$, $777 a], \theta=[\pi / 4,3 \pi / 4]$ and $\phi=[0,2 \pi]$, and a resolution of $\left[N_{r}\right.$, $\left.N_{\theta}, N_{\phi}\right]=[1152,3,768]$. The stellar and the pulsar winds have a spherical symmetry and are injected at the inner boundary $(r=0.62 a)$. The remaining boundaries have outflow conditions. The pulsar wind is injected in a sector on the orbital plane with a half-opening angle of $0.84 \mathrm{rad}$, which corresponds to a pulsar-tostellar wind thrust ratio of $\eta=\left(L_{\mathrm{sd}} / c\right) /\left(\dot{M} v_{\mathrm{w}}\right)=0.1$, and the stellar wind is injected in the remaining $\phi$-interval. We use an ideal equation of state with a polytropic index of $4 / 3$. The injection densities of the stellar and the pulsar winds were chosen so as to reproduce a pulsar-to-stellar wind thrust ratio of $\eta=0.1$; the Mach numbers of the pulsar and stellar winds at injection are 26 and 7, respectively; the stellar wind speed and the pulsar wind Lorentz factor are fixed to $v_{\mathrm{w}}=0.008 c$ and $\Gamma=3$, respectively (see e.g. Bosch-Ramon et al. 2012, 2015, regarding the validity of moderate Lorentz factors). The orientation of the pulsar-wind injection sector rotates with time reproducing the orbital motion of $T=321 \mathrm{~d}$ accounting for the eccentricity of the system $(e=0.83)$ and taking the masses of the Be star and the pulsar to be $20 \mathrm{M}_{\odot}$ and $1.44 \mathrm{M}_{\odot}$, respectively (yielding $a \approx 4 \times 10^{13} \mathrm{~cm}$ ). In the simulations, the periastron and apastron pulsar locations are to the right and to the left from the point $(0,0)$, respectively. As noted in Barkov \& Bosch-Ramon (2016), the mass of the Be disc is likely much smaller than that injected by the spherical stellar wind and is thus neglected in this study. The disc could however add some extra mass to the shocked

\footnotetext{
${ }^{2}$ Link http://plutocode.ph.unito.it/index.html
}
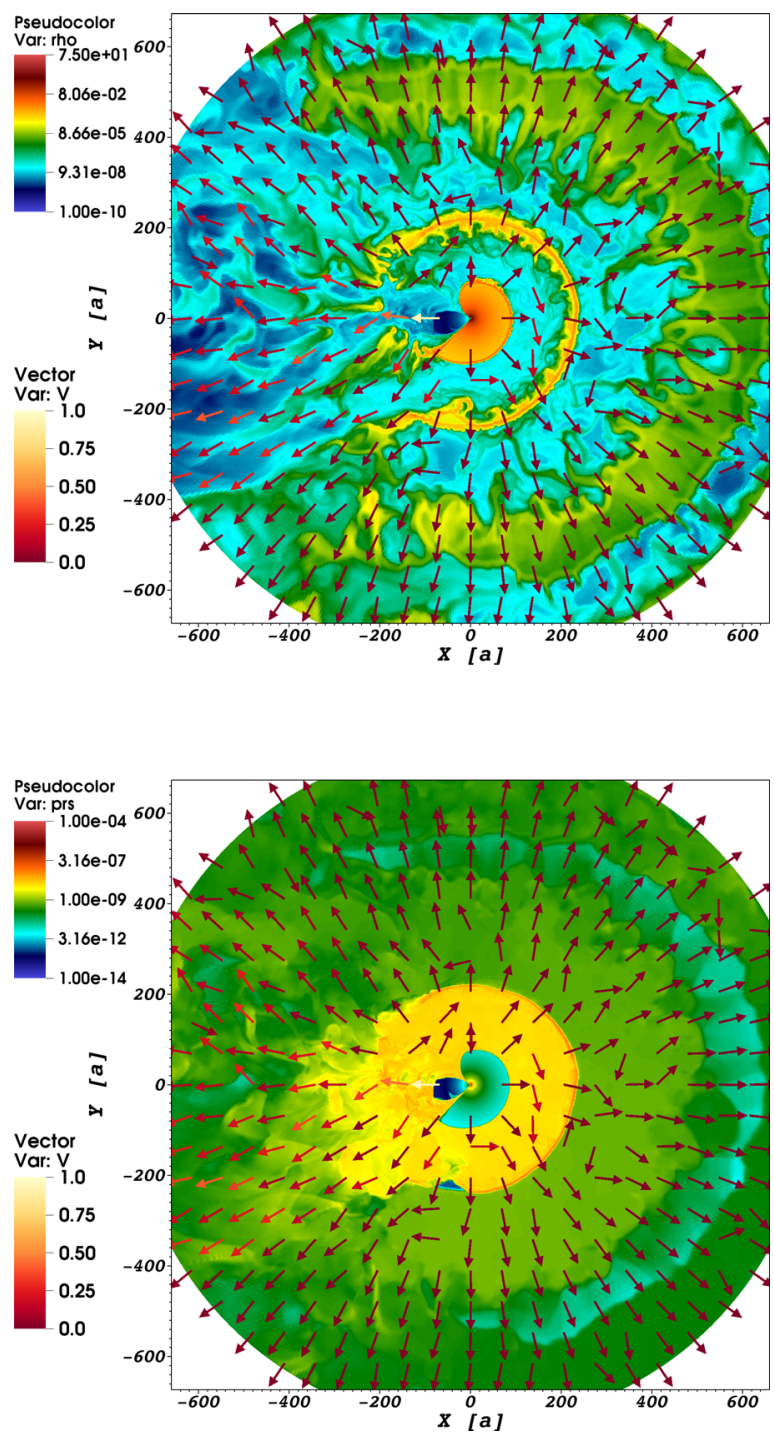

Figure 1. Colour maps of density (top panel) and pressure (bottom panel), and coloured arrows representing the flow direction and the three-velocity modulus, in the orbital plane after $1013 \mathrm{~d}$, or $210 \mathrm{~d}$ after the last periastron passage $(\psi \approx 0.65)$. The apastron direction is to the left.

winds around periastron passage, which deserves a devoted study. The simulations are run for a time $t \gtrsim 3 T(1013 \mathrm{~d})$ to eliminate the effects of the initial conditions on the results.

\subsection{Simulation results}

In Fig. 1, we plot the maps of density (top) and pressure (bottom) on the orbital plane, with coloured arrows indicating the flow direction and three-velocity modulus.

The three low-density (blue) semi-rings to the right (periastron direction) of the density map in Fig. 1, top panel, consist of shocked pulsar wind, and indicate that the pulsar has already gone through three orbits at $t=1013 \mathrm{~d}(\psi \approx 0.65)$. Intercalated with these semirings, there are larger-density (green/yellow) semi-rings that consist of shocked stellar wind. This stellar wind was injected rightwards when the pulsar was far from periastron, and the interacting twowind structure on the binary scales (i.e. the pulsar wind injector and its boundary region with the stellar wind) was directed leftwards (apastron direction). Because of the system's high eccentricity, the 


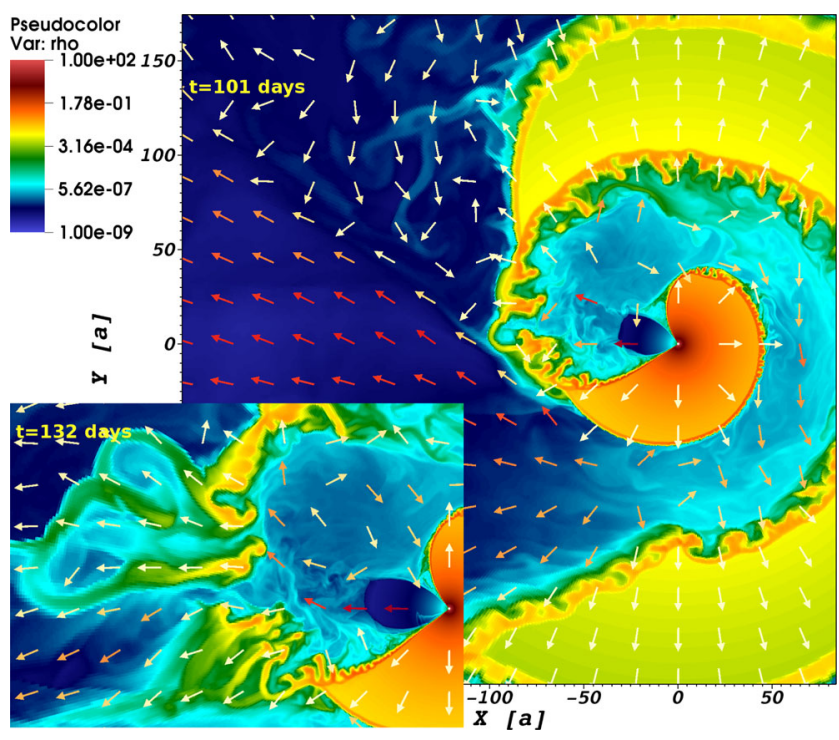

Figure 2. Colour maps of the density, with coloured arrows representing the flow direction and the three-velocity modulus, for $t=101$ and $132 \mathrm{~d}$ (inlet; same scale), i.e. $\psi \approx 0.32$ and 0.41 .

amount of stellar (pulsar) wind injected in the apastron direction is much less (more) than in the periastron direction because of the very long time spent by the pulsar far from periastron. This leads to the fast disruption $(\lesssim 1 T)$ of the shocked stellar wind semi-ring in the apastron direction produced by the impact of the pulsar wind.

The pressure map presented in Fig. 1, bottom panel, shows clearly separated pressure regions: (i) the high-pressure, inner-most yellow region, i.e. within the first turn of the spiral-like structure formed by the shocked winds, which is filled by the energy of the pulsar wind injected during the transit from periastron to apastron and (ii) the lower-pressure, green region, outside the first turn of the spiral, where a negative, smooth pressure gradient leads to gradual shocked flow acceleration. The outflow in the apastron direction, mostly supersonic, is splattered by shocks, whereas in the periastron direction, the flow intercalates regions of subsonic shocked pulsar wind and shocked (but supersonic) stellar wind. ${ }^{3}$

The energy accumulated in the yellow inner-most region of the pressure map abruptly drops around apastron, when the escape rate of shocked pulsar wind from this region suddenly increases. The apastron release of energy accumulated in the first turn of the spiral is caused by the disruption of the stellar wind semi-ring, which has confined the shocked pulsar wind in the apastron side during the transit from periastron to apastron. This disruption process is illustrated by Fig. 2, which shows the colour map of the density for $t=101(\psi \approx 0.32)$ and $132 \mathrm{~d}(\psi \approx 0.41$; inlet, same scale $)$, for a region close to the binary around apastron. The drop in energy within the inner-most region is clearly visible in the evolution of the total internal energy within $r=100 a$, shown in Fig. 3. Given the low resolution in $\theta$, the result must be considered qualitatively, although the predicted strong modulation is compelling.

The typical radial velocity $\left(v_{\mathrm{r}}\right)$ of the flows moving in the direction of apastron $(\mathrm{Sa})$, periastron $(\mathrm{Sp})$ and normal to the apastronperiastron axis $(\mathrm{Sn})$ can be computed by integrating the product of $v_{\mathrm{r}}$ with $\rho$ over some $\phi$ interval on the orbital plane $(\theta=0)$ : $\left\langle v_{\mathrm{r}}\right\rangle=\int_{\phi_{b}}^{\phi_{e}} \rho v_{\mathrm{r}} d \phi / \int_{\phi_{b}}^{\phi_{e}} \rho d \phi$.

\footnotetext{
${ }^{3}$ The flows are sub- or supersonic with respect to their own sound speed.
}

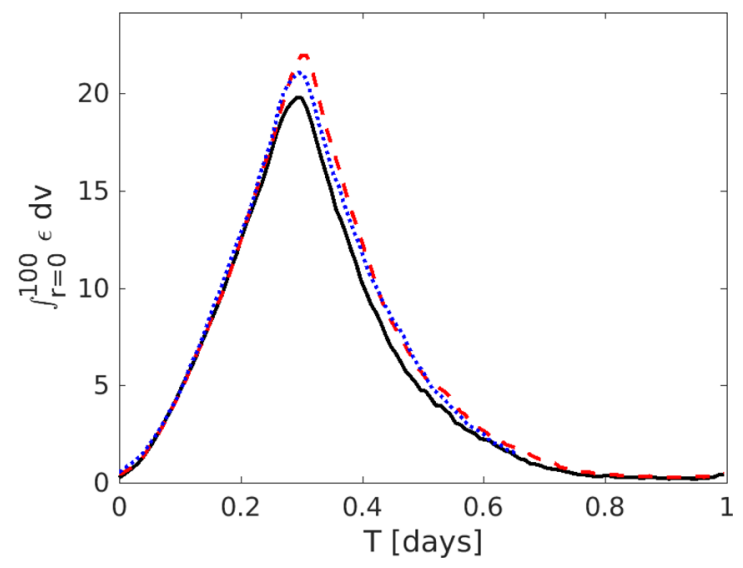

Figure 3. Orbital evolution of the total internal energy accumulated within $r=100 a$, in arbitrary units, for three orbits (orbit 1: solid line; orbit 2: long dashed line; orbit 3: dotted line).

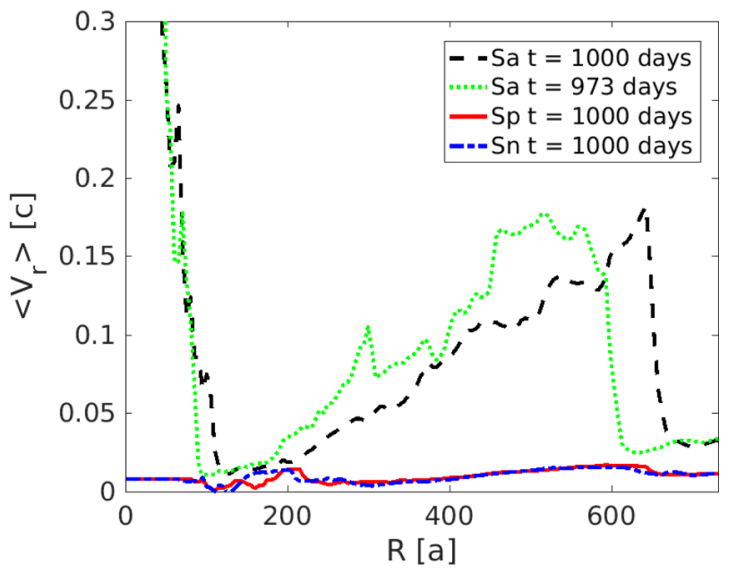

Figure 4. Density-weighted radial velocity distribution with $r$ for different directions (Sa, Sp and $\mathrm{Sn})$ for $t=973$ and $1000 \mathrm{~d}(\psi \approx 0.53,0.62)$.

The sectors $\mathrm{Sa}, \mathrm{Sp}$ and $\mathrm{Sn}$ are chosen to illustrate the typical flow $\left\langle v_{\mathrm{r}}\right\rangle$-values in different directions away from the binary, and correspond to the $\phi$-ranges $\left[\phi_{b}, \phi_{e}\right]=[2.62,3.66],\left[\phi_{b}, \phi_{e}\right]=[5.07$, $6.12]$ and $\left[\phi_{b}, \phi_{e}\right]=[1.05,2.09]$, respectively. The angular intervals are equal and $\phi$ grows counterclockwise from periastron.

The $r$-distribution of $\left\langle v_{\mathrm{r}}\right\rangle$ in the $\mathrm{Sa}, \mathrm{Sp}$ and $\mathrm{Sn}$ directions is presented in Fig. 4 for $t=1000 \mathrm{~d}(\psi \approx 0.62)$, showing also $t=973 \mathrm{~d}(\psi$ $\approx 0.53$ ) for Sa to illustrate the smoothness of the $r$-distribution of $\left\langle v_{\mathrm{r}}\right\rangle$ in that direction. The figure shows that $\left\langle v_{\mathrm{r}}\right\rangle$ does not change much in absolute terms $(\sim 0.01 c)$ in the $\mathrm{Sp}$ and $\mathrm{Sn}$ directions, whereas in the Sa direction $v_{\mathrm{r}}$ quickly rises reaching $\left\langle v_{\mathrm{r}}\right\rangle \sim 0.15 c$ already at $r \sim 600 a \approx 2 \times 10^{16} \mathrm{~cm}$.

Finally, Fig. 5 presents the colour maps of the $\left\langle v_{\mathrm{r}}\right\rangle$ dependence on $t$ and $r$ for the Sp (top) and Sa (bottom) directions. These maps are $2 \mathrm{D}$ space-time representations of $\left\langle v_{\mathrm{r}}\right\rangle$, which allows one to track the $t$-evolution of the location of $\left\langle v_{\mathrm{r}}\right\rangle$ jumps along $r$ indicating a general flow acceleration. In addition, one can see in the figure that at fixed $t$-values there are sudden drops in $\left\langle v_{\mathrm{r}}\right\rangle$ along $r$ which indicate the presence of front shocks. Because of the presence of the heavy stellar wind semi-rings, at the radius $r=500$ a in the Sp direction, $\left\langle v_{\mathrm{r}}\right\rangle$ is just $\sim 0.02 c$, showing time variations caused by the arrival of the intercalated shells of shocked stellar and pulsar wind. On the other hand, in the Sa direction, the flow reaches mildly relativistic radial velocities, $\left\langle v_{\mathrm{r}}\right\rangle \sim 0.05-0.2 c$, with recurrent time 


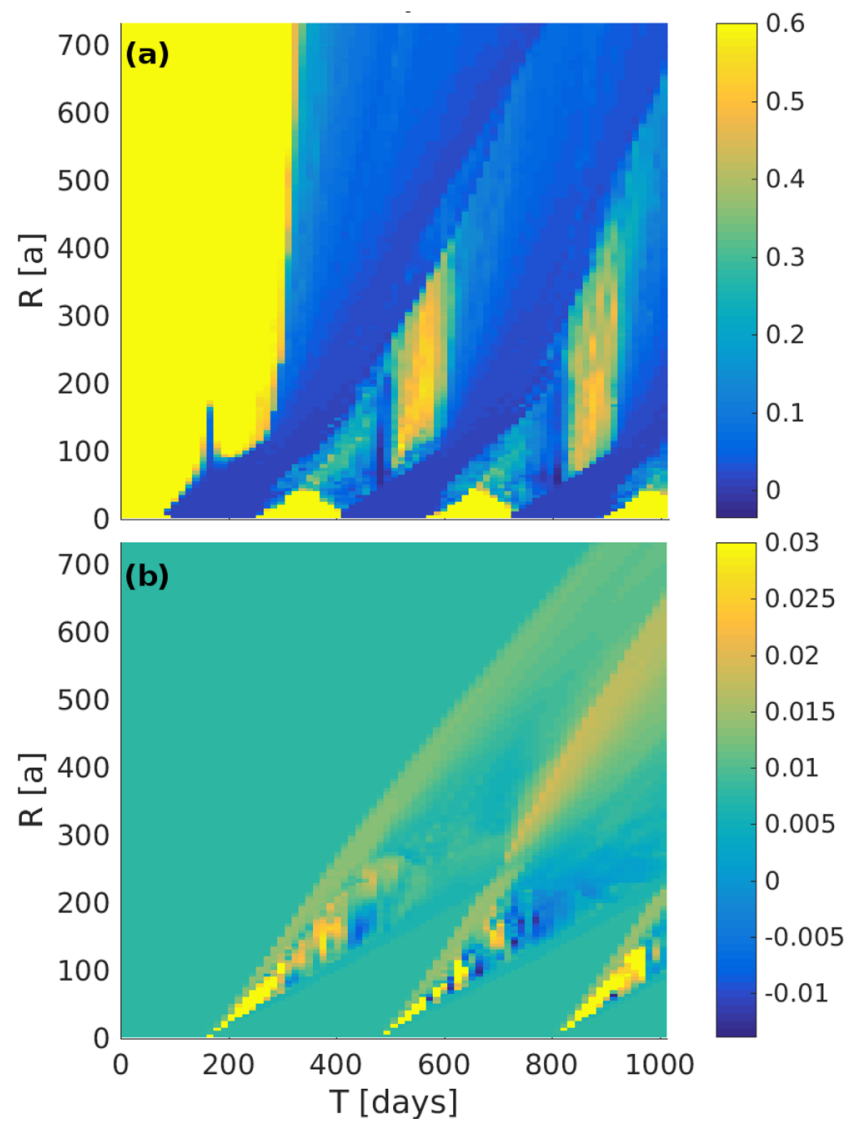

Figure 5. Colour maps showing the density-weighted radial velocity dependence on $t$ and $r$ for different directions: Sp (top) and Sa (bottom).

variations produced by the irregular disruption of the stellar wind semi-ring and subsequent pulsar wind mass loading. At $r=500 a$, the system can be considered approximately in a steady state for $t$ $\gtrsim 400-500 \mathrm{~d}$ in the Sa direction, and $t \gtrsim 600-700 \mathrm{~d}$ in the Sp and Sn directions.

\section{DISCUSSION AND SUMMARY}

As described in Section 1, the X-ray, GeV and VHE light curves of HESS J0632+057 have a maximum before apastron, around $\psi \sim 0.3-0.4$, and a fast drop around $\psi \sim 0.4-0.5$, and the X-ray and VHE light curves have a secondary, less prominent maximum around $\psi \sim 0.6-0.8$ (Aliu et al. 2014). In the context of this work, the X-ray, $\mathrm{GeV}$ and VHE emission rise around $\psi \sim 0.3-0.4$ would be related to the growing energy injected by the pulsar wind after periastron passage. In the leptonic scenario (X-rays: synchrotron, $\mathrm{GeV}$ and VHE: inverse Compton (IC); the most efficient in the present context), this energy increase would compensate the decrease in IC emission due to a larger distance from the star. The drop in $\mathrm{X}$-ray, $\mathrm{GeV}$ and VHE emission would be mainly produced then by the efficient removal of energy from this region around $\psi \gtrsim 0.4$, when the stellar wind semi-ring in the apastron direction is disrupted and then blown away by the pulsar wind impact. All this is clearly illustrated by the evolution of the total internal energy accumulated within $100 a$, shown in Fig. 3 .

Given the typical size of the region, $\sim 10^{15} \mathrm{~cm}$, and the stellar luminosity, $\sim 10^{38} \mathrm{erg} \mathrm{s}^{-1}$, the IC cooling time-scale for TeV electrons is $\sim 10^{8} \mathrm{~s}$ (Khangulyan, Aharonian \& Kelner 2014). At the typical speeds involved on those scales, say $\sim 0.03 c \approx 10^{9} \mathrm{~cm} \mathrm{~s}^{-1}$, the potentially present $\mathrm{TeV}$ electrons emitting $\mathrm{X}$ - and VHE gamma-rays would radiate $\sim 1$ per cent of their energy before leaving the region. Given the modest X-ray and VHE luminosities, of $\sim 10^{33} \mathrm{erg} \mathrm{s}^{-1}$, this implies an electron energy injection rate of $\sim 10^{35} \mathrm{erg} \mathrm{s}^{-1}$, compatible with a pulsar spin-down power similar for instance to that of PSR B1259-63 $\left(\approx 8 \times 10^{35} \mathrm{erg} \mathrm{s}^{-1} \text {; Johnston et al. 1992 }\right)^{4}$. A large magnetic field in the emitting region may require a larger energy budget to explain the VHE luminosities through IC because of the competition between IC and synchrotron losses. Nevertheless, although an accurate assessment of the relation between X-ray and the VHE emitting electrons needs specific modelling, the similar X-ray and VHE fluxes observed already imply that synchrotron losses cannot be much stronger than IC losses for those electrons (Aliu et al. 2014). The origin of the second, less prominent maximum around $\psi \sim 0.6-0.8$ cannot be derived from the simulation results in a straightforward way, and a detailed study is needed to consistently include the second maximum in the picture just described.

The weak radio emission seen in VLBI, with fluxes $\sim 0.2$ $0.5 \mathrm{mJy}$, can be explained by the synchrotron emission in radio of the same population of X-ray, $\mathrm{GeV}$ and VHE emitting electrons, although a non-thermal model encompassing the radio, $\mathrm{X}$-ray, $\mathrm{GeV}$ and VHE emission is required to further check the consistency of this scenario. With this caveat in mind, one can still propose that the shift in position of the core emission, and the decrease in flux seen by VLBI observations (Moldón et al. 2011) around $\psi \sim 0.4$, can be explained by the disruption of the apastron side stellar semiring starting around that orbital phase. In parallel with this process, the radio emitting region, associated with the inner-most semi-ring of shocked pulsar wind on the periastron side (see Fig. 2), would become more prominent, shifting the core location of the source towards periastron. In this scenario, the impact between the pulsar wind and the fragments of stellar wind in the apastron direction could explain the radio component extended in that direction (see Barkov \& Bosch-Ramon 2016, for a similar explanation regarding the X-ray extended emission in PSR B1259-63). The size of the region highlighted by Fig. 2 is $\sim 100 a$, whereas the observed radio emitting structures are $\sim 25 a$ in the projected distance, but we indicate that: (i) the de-projected size may be easily larger by a factor of two and (ii) the values of $\eta$ and wind speed are poorly constrained, leaving room to accommodate another factor of $\sim 2$ difference between the simulated and the actual radio source size.

The stellar wind fragments released around apastron passage in the apastron direction could be detected as extended X-ray emitting structures, as proposed by Barkov \& Bosch-Ramon (2016) for those seen in PSR B1259-63 by Pavlov et al. (2015). In the case of HESS J0632+057, the orbital period is $\approx 4$ times smaller, but the source is at a similar galactic distance. Since the extended Xray emission size is $\propto T$, as this is the growth time-scale of the structure, it is somewhat harder to resolve this X-ray component for HESS J0632+057 than for PSR B1259-63 from Earth. The spin-down power of the putative pulsar in HESS J0632+057 is also unknown, and a lower value than in PSR B1259-63 would make the emission fainter. On the other hand, the orbital period is $\approx 4$ times shorter, which leads to more frequent stellar wind fragment release than in the case of PSR B1259-63, leading to a more continuous structure. For all this, we propose that arcsecond scale extended emission from HESS J0632+057, slightly more compact than in

\footnotetext{
${ }^{4}$ The energetic requirements in $\mathrm{GeV}$ ( $\mathrm{Li}$ et al. 2017) are similar to those in $\mathrm{X}$-rays and VHE.
} 
PSR B1259-63, could potentially be detected with Chandra for an exposure similar to that of PSR B1259-63 Chandra observations (Pavlov et al. 2015).

We conclude that the simulations presented here provide with a robust, semi quantitative description of the pulsar-stellar wind interaction along the orbit in a strongly eccentric system such as HESS J0632+057. They allow us to envision a broad picture that can qualitatively explain most of the non-thermal phenomenology in this source.

Three-dimensional simulations with a higher resolution in the $\theta$-direction, also including the magnetic field at a later stage, are planned for a more accurate study of the fluid dynamics, and detailed modelling of the non-thermal emission is also on the way.

\section{ACKNOWLEDGEMENTS}

We want to thank the referee for a constructive and useful report. The calculations were carried out in the CFCA cluster of National Astronomical Observatory of Japan and GreatWave FX100 Fujitsu cluster in RIKEN. We thank the PLUTO team for the possibility to use the PLUTO code and for technical support. The visualization of the results is performed in the VisIt package (Hank Childs et al. 2012). This work was supported by the Spanish Ministerio de Economía y Competitividad (MINECO/FEDER, UE) under grants AYA2013-47447-C3-1-P and AYA2016-76012-C3-1-P with partial support by the European Regional Development Fund (ERDF/FEDER), MDM-20140369 of ICCUB (Unidad de Excelencia 'María de Maeztu'), and the Catalan DEC grant 2014 SGR 86. This research has been supported by the Marie Curie Career Integration Grant 321520. The project was also supported by the JSPS (Japan Society for the Promotion of Science): Nos 2503786, 25610056, 26287056 and 26800159, NSF grant AST-1306672 and DoE grant DESC0016369. VBR acknowledges financial support from MINECO and European Social Funds through a Ramón y Cajal fellowship. BMV acknowledges MEXT (Ministry of Education, Culture, Sports, Science and Technology of Japan): No. 26105521. BMV acknowledges partial support from the NSF grant AST1306672 and DoE grant DE-SC0016369.

\section{REFERENCES}

Aharonian F. et al., 2005a, A\&A, 442, 1

Aharonian F. et al., 2005b, Science, 309, 746

Aharonian F. A. et al., 2007, A\&A, 469, L1

Albert J. et al., 2006, Science, 312, 1771

Aliu E. et al., 2014, ApJ, 780, 168

Barkov M. V., Bosch-Ramon V., 2016, MNRAS, 456, L64

Bongiorno S. D., Falcone A. D., Stroh M., Holder J., Skilton J. L., Hinton J. A., Gehrels N., Grube J., 2011, ApJ, 737, L11

Bosch-Ramon V., Barkov M. V., Khangulyan D., Perucho M., 2012, A\&A, 544, A59

Bosch-Ramon V., Barkov M. V., Perucho M., 2015, A\&A, 577, A89

Casares J., Ribó M., Ribas I., Paredes J. M., Vilardell F., Negueruela I., 2012, MNRAS, 421, 1103

Dubus G., 2013, A\&AR, 21, 64

Hank Childs H. et al., 2012, in,Wes Bethel E., Childs H., Hansen C., eds, High Performance Visualization-Enabling Extreme-Scale Scientific Insight, Chapman \& Hall/CRC Computational Science, Taylor \& Francis, p. 357

Hinton J. A. et al., 2009, ApJ, 690, L101

Johnston S., Manchester R. N., Lyne A. G., Bailes M., Kaspi V. M., Qiao G., D’Amico N., 1992, ApJ, 387, L37

Kargaltsev O., Pavlov G. G., Durant M., Volkov I., Hare J., 2014, ApJ, 784, 124

Khangulyan D., Aharonian F. A., Kelner S. R., 2014, ApJ, 783, 100

Li J., Torres D. F., Cheng K.-S., de Ona Wilhelmi E., Kretschmar P., Hou X., Takata J., 2017, ApJ, preprint (arXiv:1707.04280)

Mignone A., Bodo G., 2005, MNRAS, 364, 126

Mignone A., Bodo G., Massaglia S., Matsakos T., Tesileanu O., Zanni C., Ferrari A., 2007, ApJS, 170, 228

Moldón J., Ribó M., Paredes J. M., 2011, A\&A, 533, L7

Paredes J. M., Martí J., Ribó M., Massi M., 2000, Science, 288, 2340

Pavlov G. G., Hare J., Kargaltsev O., Rangelov B., Durant M., 2015, ApJ, 806, 192

Tavani M., Kniffen D., Mattox J. R., Paredes J. M., Foster R. S., 1998, ApJ, 497, L89

Zamanov R. K., Marti J., Garcia-Hernandez M. T., 2017, preprint (arXiv:1702.06947)

This paper has been typeset from a $\mathrm{T}_{\mathrm{E}} \mathrm{X} / \mathrm{L} \mathrm{T} \mathrm{E} X$ file prepared by the author. 